\title{
The P66Shc/Mitochondrial Permeability Transition Pore Pathway Determines Neurodegeneration
}

\author{
Costanza Savino, PierGiuseppe Pelicci, and Marco Giorgio \\ Department of Experimental Oncology, European Institute of Oncology, Via Adamello 16, 20139 Milan, Italy \\ Correspondence should be addressed to Marco Giorgio; marco.giorgio@ieo.eu
}

Received 13 February 2013; Revised 19 April 2013; Accepted 24 April 2013

Academic Editor: Renata Santos

Copyright ( 2013 Costanza Savino et al. This is an open access article distributed under the Creative Commons Attribution License, which permits unrestricted use, distribution, and reproduction in any medium, provided the original work is properly cited.

\begin{abstract}
Mitochondrial-mediated oxidative stress and apoptosis play a crucial role in neurodegenerative disease and aging. Both mitochondrial permeability transition (PT) and swelling of mitochondria have been involved in neurodegeneration. Indeed, knockout mice for cyclophilin-D (Cyc-D), a key regulatory component of the PT pore (PTP) that triggers mitochondrial swelling, resulted to be protected in preclinical models of multiple sclerosis (MS), Parkinson's disease (PD), and amyotrophic lateral sclerosis (ALS). However, how neuronal stress is transduced into mitochondrial oxidative stress and swelling is unclear. Recently, the aging determinant p66Shc that generates $\mathrm{H}_{2} \mathrm{O}_{2}$ reacting with cytochrome $\mathrm{c}$ and induces oxidation of PTP and mitochondrial swelling was found to be involved in MS and ALS. To investigate the role of p66Shc/PTP pathway in neurodegeneration, we performed experimental autoimmune encephalomyelitis (EAE) experiments in p66Shc knockout mice (p66Shc-l-), knock out mice for cyclophilin-D (Cyc-D-/-), and p66Shc Cyc-D double knock out (p66Shc/Cyc-D-/-) mice. Results confirm that deletion of p66Shc protects from EAE without affecting immune response, whereas it is not epistatic to the Cyc-D mutation. These findings demonstrate that p66Shc contributes to EAE induced neuronal damage most likely through the opening of PTP suggesting that p66Shc/PTP pathway transduces neurodegenerative stresses.
\end{abstract}

\section{Introduction}

The p66Shc protein is the largest isoform encoded by the ShcA locus located in the chromosome 1 in the human or chromosome 3 in the mouse genome. The ShcA locus encodes three isoforms through two different promoters, one for the p46 and p52 isoforms and the other one for the p66 isoform. Notably, the p66Shc isoform is peculiar of vertebrates being conserved in Fucu, Xenophus, Rattus, Mus, and Homo but not in Saccharomyces, Caenorhabditis, and Drosophila [1]. The p52Shc and p46Shc function as adaptor protein in signal transduction pathways linking different activated receptor tyrosine kinases to the Ras pathway. P66Shc is not involved in Ras activation, although p66Shc has the typical domain organization of all members of the Shc family of adaptor proteins. Instead, p66Shc functions in the intracellular pathways that convert oxidative signals into apoptosis $[2,3]$.

A fraction of p66Shc has been observed within the mitochondrial intermembrane space [4], and in vitro experiments revealed that the recombinant $\mathrm{p} 66 \mathrm{Sh}$ c human protein interacts and oxidizes $\mathrm{Fe}^{2+}$ cytochrome $\mathrm{c}$ to form $\mathrm{H}_{2} \mathrm{O}_{2}$ through a redox center that has been mapped to the $\mathrm{N}$-terminus of the p66Shc protein and is missing in the other two ShcA isoforms [5]. Thus, mitochondrial p66Shc sequesters electrons from the respiratory chain to generate reactive oxygen species (ROS).

P66Shc function is tightly regulated at multiple levels. First of all the total amount of p66Shc is regulated by transcriptional [6] and posttranslational mechanisms. In particular, the half-life of p66Shc has been demonstrated to increase upon apoptotic stimulation, notably in a p53dependent manner [7]. The localization of p66Shc into mitochondrial intermembrane space is regulated by p66Shc posttranslational modifications including serine phosphorylation by stress kinases like Jnk-1 and Pkc-B and prolylisomerization by Pin-1, that induce p66Shc translocation within mitochondria [8].

A further level of activation of p66Shc mitochondrial function is represented by the availability of unbound 
p66Shc within mitochondrial vesicles. In fact, mitochondrial p66Shc has been observed to associate with a high molecular weight complex of about $670 \mathrm{KDa}$ and to the mitochondrial chaperon mtHsp70. Notably, treatment of cells with proapoptotic stimuli such as Ultraviolet radiation or $\mathrm{H}_{2} \mathrm{O}_{2}$ induces the dissociation of this complex and thus the release of monomeric p66Shc free to react with cytochrome c [9]. Finally, the oxidation of cysteine residues and oligomerization state of p66Shc have been reported to regulate its redox function within mitochondria [10].

The activation of p66Shc, and the consequent $\mathrm{H}_{2} \mathrm{O}_{2}$ accumulation in the mitochondria, impacts on the integrity of mitochondrial inner membrane and transmembrane potential [9]. The addition of recombinant p66Shc protein to isolated mouse liver mitochondria is sufficient to induce opening of the PTP and ballooning of the vesicles [5]. The key event leading to the mitochondrial swelling is indeed the opening of the permeability transition pore (PTP), a highconductance inner membrane channel whose molecular components have not been identified, that triggers the inner membrane permeability to solutes and as consequence to water [11].

These events are central node of cell death. During apoptosis mitochondria undergo structural and functional remodeling leading to the swelling of the organelles with the subsequent release of apoptogenic factors including cytochrome c, Smac/DIABLO, AIF, and Omi/HtrA2 into the cytosol. These factors activate a cascade of proteases responsible for nuclear DNA fragmentation and finally cell death [12]. The PTP open-closed state is regulated by multiple effectors that act on various sites [13]. In particular, it has been shown that reactive oxygen species producing by mitochondrial respiration are key regulators of PTP opening [14].

Consistently with the role proposed for oxidative stress on cell death and aging [15], primary mouse embryonic fibroblasts (MEFs) derived from p66Shc-/- embryos have lower intracellular concentration of ROS, as revealed by the reduced oxidation of ROS-sensitive probes and the reduced accumulation of endogenous markers of oxidative stress (8-oxo-guanosine) [7]. Likewise, p66Shc-/- mice have diminished levels of both systemic isoprostane [16] and intracellular (nitrotyrosines, 8-oxo-guanosine) oxidative stress [7].

Moreover, p66Shc-/- cells were shown to be resistant to apoptosis induced by a variety of different signals, including $\mathrm{H}_{2} \mathrm{O}_{2}$, UV, staurosporine, taxol, growth factor deprivation, calcium ionophore, osmotic shock, and CD3-CD4 crosslinking [2], and similarly different tissues of the p66Shc-/mice were found to be resistant to apoptosis induced by paraquat [17], hypercholesterolemia [16], hyperglycemia [18], immunotoxicity [19], and ischemia [20].

Finally it is not surprising that p66Shc-/- mice resulted to be protected from aging-associated diseases, such as metabolic syndrome [20,21] atherosclerosis [16], diabetes [18], and neurodegeneration $[19,22,23]$ and show prolonged life span $[17,21,22]$, and p66Shc/PTP pathway appears a crucial pathway of stress response involved in tissue dysfunction.

\section{Materials and Methods}

2.1. Cells. NG108-15, N2A, and the SH-SY5Y were grown at $37^{\circ} \mathrm{C}$ in $5 \% \mathrm{CO}_{2}$ in Dulbecco's modified Eagle's medium (DMEM) the NG108-15, Modified Eagle's medium (MEM) the N2A, and the SH-SY5Y with F12 1:1 and 1\% NeAA, supplemented with $10 \%$ (or $15 \%$ the SH-SY5Y) fetal bovine serum S.A. (Invitrogen, Carlsbad, CA, USA) and penicillinstreptomycin and glutamine (100 units/mL, $100 \mu \mathrm{g} / \mathrm{mL}$ ) (Invitrogen).

Kelly and PC12 were cultured in RPMI 1640 medium (Gibco-BRL, Eggenstein, Germany) supplemented with 10\% FBS (and 5\% Horse serum the PC12) and penicillinstreptomycin (100 units $/ \mathrm{mL}, 100 \mu \mathrm{g} / \mathrm{mL}$ ) (Invitrogen), under $5 \% \mathrm{CO}_{2}$ at $37^{\circ} \mathrm{C}$.

2.2. Western Blotting. Cells were washed twice with icecold PBS and lysed with $200 \mu \mathrm{L}$ of lysis buffer. Lysates were vortexed and incubated on ice for $15 \mathrm{~min}$ twice and then cleared by spinning at $13.000 \mathrm{rpm}$ for $5 \mathrm{~min}$ at $4^{\circ} \mathrm{C}$. Proteins were separated by $10 \%$ SDS-PAGE gels and immunoblotted according to standard western blotting procedures using primary antibody (polyclonal anti-shc, BD Biosciences).

2.3. Mice. P66Shc-/-, Cyc-D-/-, and p66Shc/Cyc-D-/mice were bred in the certified IFOM-IEO campus animal facility in accordance with national and institutional guidelines.

Mice were housed in an air-conditioned room (temperature $21 \pm 1^{\circ} \mathrm{C}$, relative humidity $60 \pm 10 \%$ ) with a whitered light cycle (lights on from 07:00 to 19:00) and with ad libitum food availability (2018S Teklad Global 18\% Protein Rodent Diet, provided by Harlan Teklad) and drinking water (autoclaved tap water).

All the in vivo experiments were performed in accordance with Italian laws and regulations.

2.4. EAE. EAE was induced in p66Shc-/-, Cyc-D-/-, p66Shc/Cyc-D-/-, and C57BL/6 (Wilde type, WT) female mice (6-8 weeks of age). WT mice were obtained from Charles River (Calco, Italy) and housed in specific pathogenfree conditions, allowing access to food and water ad libitum. Procedures involving animals and their care were conducted in conformity with the institutional guidelines in compliance with national (D.L. n. 116, G.U., suppl. 40, February 18, 1992) and international laws and policies (EU Council Directive 86/609, OJ L 358, 1, December 12, 1987; Guide for the Care and Use of Laboratory Animals, U.S. National Research Council, 1996). The protocols for the proposed investigation were reviewed and approved by the Animal of the European Institute of Oncology. EAE was induced by subcutaneous immunization in the flanks with a total of $200 \mathrm{Ag}$ of MOG3555 (Multiple Peptide Systems, San Diego, CA, USA) in incomplete Freund's adjuvant (Sigma, St. Louis, MO, USA) supplemented with $8 \mathrm{mg} / \mathrm{mL}$ of Mycobacterium tuberculosis (strain H37RA; Difco, Detroit, MI, USA).

Mice received $500 \mathrm{ng}$ of pertussin toxin (Sigma) i.v. at the time of immunization and $48 \mathrm{~h}$ later. Weight and clinical 


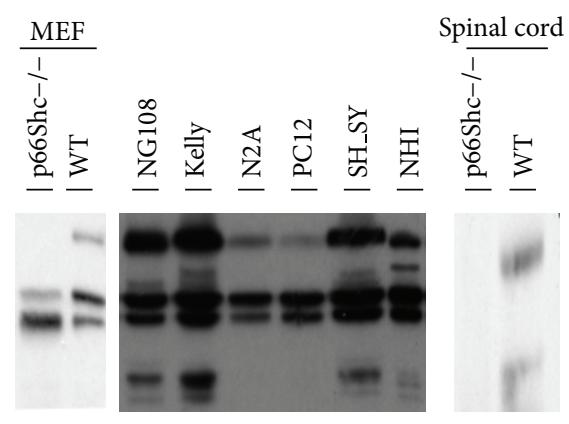

(a)

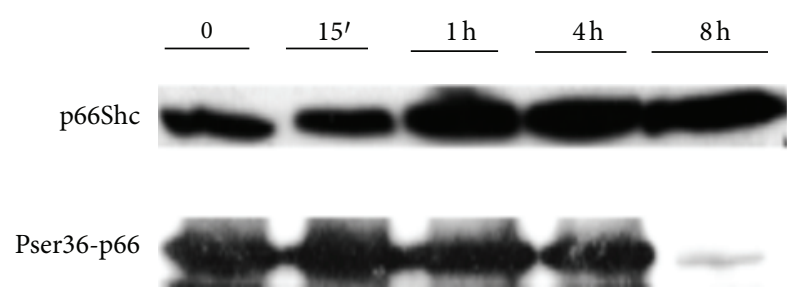

(b)
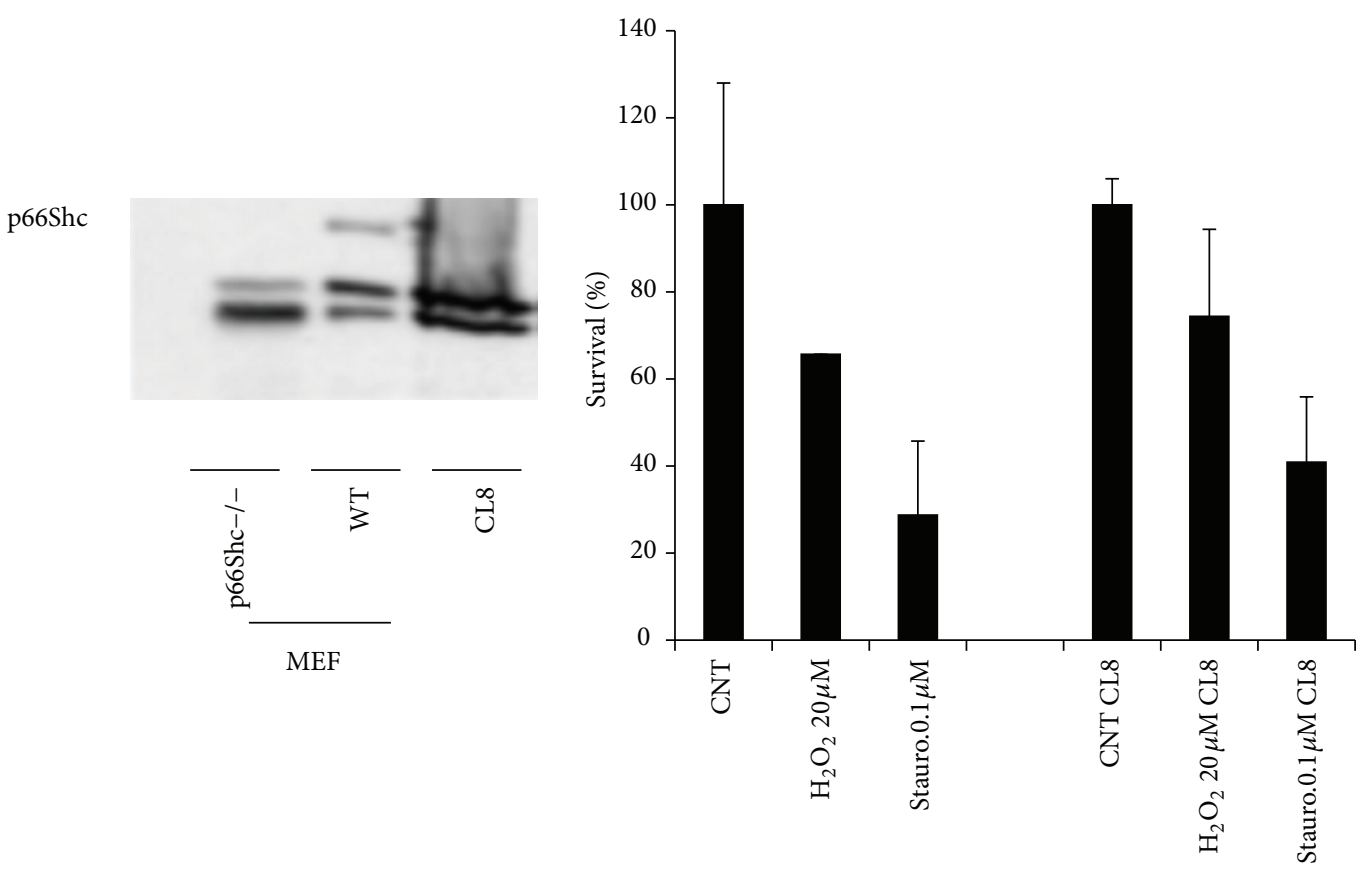

(c)

Figure 1: (a) P66Shc expression in different cell lines and spinal cord tissue. Western blot analysis of p66Shc was performed on wholecell extracts from primary mouse embryonic fibroblasts (MEF), NG108, Kelly (human neuroblastoma), N2A (mouse), PC12(rat), SH-SY5Y (human neuroblastoma), NIH cells lines, and protein extract from mouse spinal cord. (b) Expression of p66Shc (upper panel) after UV treatment and (lower panel) levels of PhopsorylatedSer36-p66Shc in Kelly cells. (c) Expression of p66Shc upon specific p66Shc RNAi Interference on Kelly and response $20 \mu \mathrm{M} \mathrm{H}_{2} \mathrm{O}_{2}$ and $0.1 \mathrm{mM}$ Staurosporine.

score were recorded daily $(0=$ healthy, $1=$ flaccid tail, $2=$ ataxia, and/or hind-limbs paresis, or slow righting reflex, 3 = paralysis of hind limb and/or paresis of forelimbs, $4=$ paraparesis of fore limb, and $5=$ moribund or death). The food pellets and the drinking water were placed on Petri plates on the floor of the cage to enable sick mice to eat and drink.

2.5. ELISA. Anti-MOG $\mathrm{MO}_{35-55}$ antibody titers were examined in the sera of the same animal groups as described previously at day 10 after immunization. Blood from these animals was obtained by heart puncture. Sera were collected and applied to $\mathrm{MOG}_{35-55}$-coated maxisorb 96-well ELISA plates (Nalge Nunc International, Rochester, NY) in serial dilutions. The plates were incubated for $1 \mathrm{~h}$ at room temperature and washed four times, and $60 \mu \mathrm{L}$ of $1: 2500$ diluted [HRP] $\alpha$-mouse
(Amersham) peroxidase conjugated mouse anti-IgG were applied to the wells. The plates were incubated for $40 \mathrm{~min}$ at room temperature and washed three times, and $60 \mu \mathrm{L}$ of ABTS $+\mathrm{H}_{2} \mathrm{O}_{2}$ substrate were applied to the wells. The reaction was performed at room temperature for $30 \mathrm{~min}$ in the dark. The level of reaction product was assessed as optic density (OD) at $415 \mathrm{~nm}$ on a standard plate reader, and the data were presented as mean_SD. OD with $n=3$ independent measurements per group.

IL-6, IFN- $\gamma$, and TNF- $\alpha$ were measured in supernatants of splenocytes (see the following) by ELISA (R\&D Systems, Inc., Minneapolis, MN, USA) according to the manufacturers' instructions.

2.6. Proliferation Assay. Cells from spleens of $\mathrm{p} 66 \mathrm{Shc}-1-$ and WT mice, stimulated or not with MOG, were cocultured for 


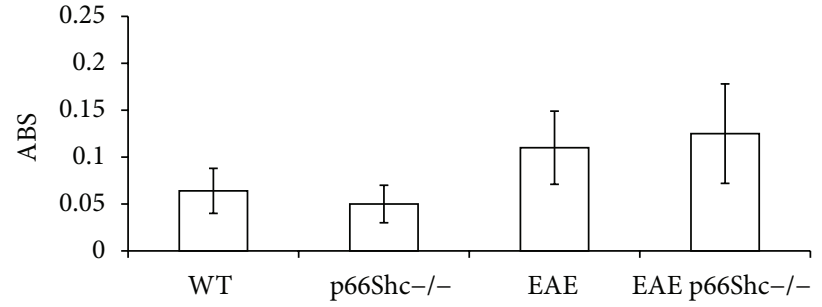

(a)

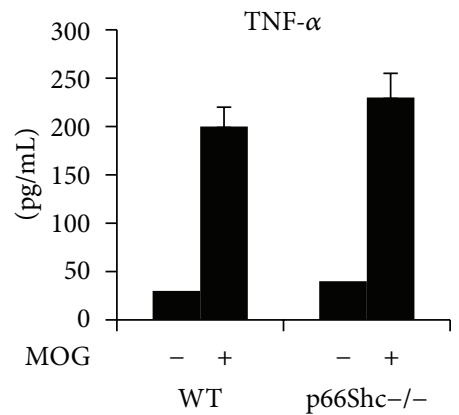

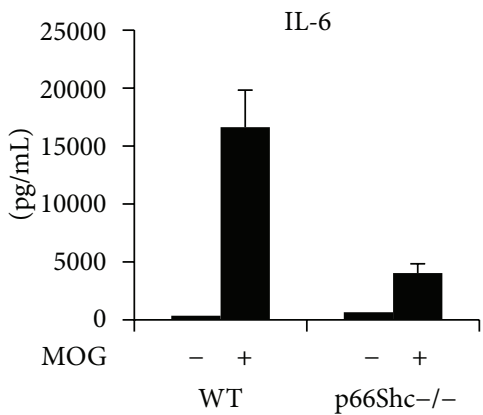

(c)

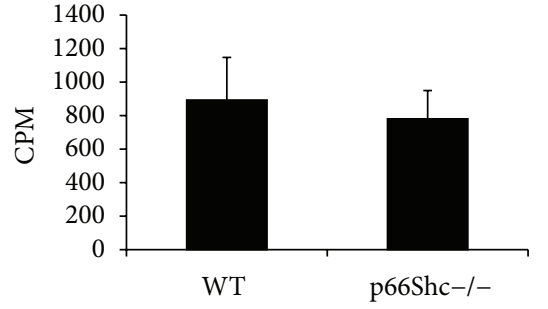

(b)

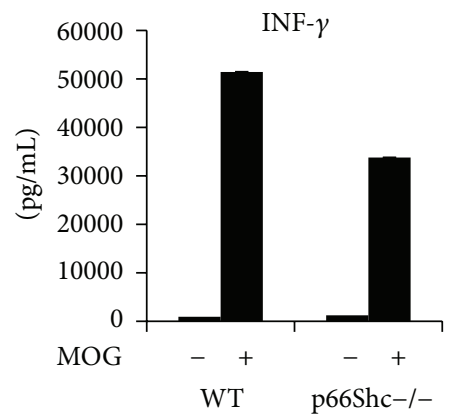

Figure 2: (a) ELISA assay for anti-MOG35-55 antibodies. (b) Proliferation assay. Cells from spleens, stimulated with MOG, were cocultured for $48 \mathrm{~h}$ and were radiolabelled with $9,25 \mathrm{kBq}$ well-1 of methyl-3H thymidine in the last $16 \mathrm{~h}$ of coculture. The cells are harvested and counted in a liquid scintillation counter. (c) Cytokines production in medium of splenocytes from WT and p66Shc-l- mice. Results are representative of one of two experiments, each with five mice per group.

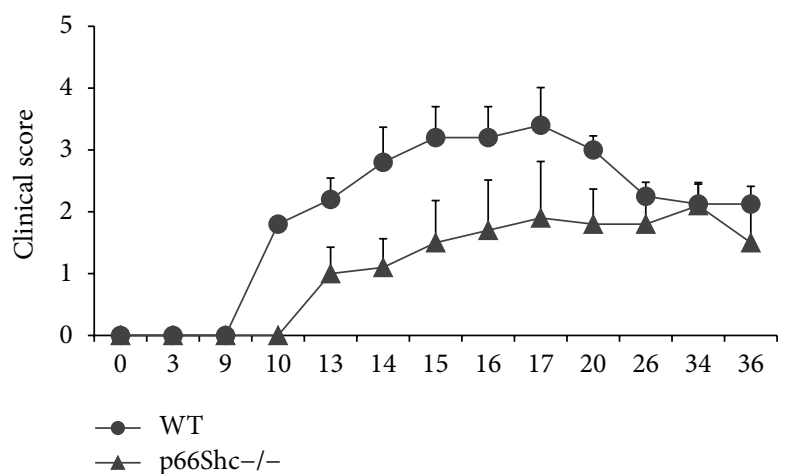

(a)

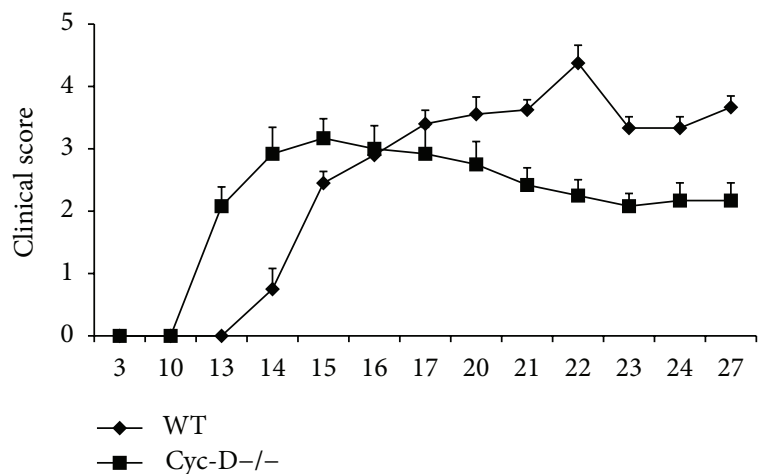

(b)

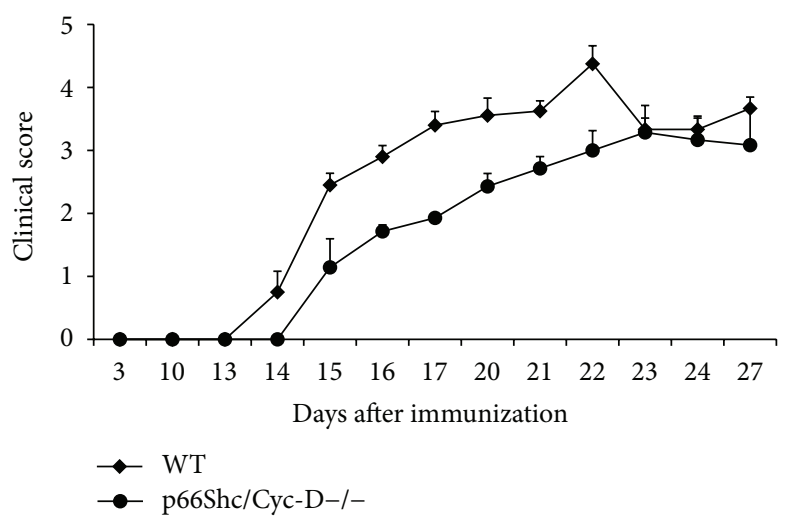

(c)

FIGURE 3: (a) EAE disease signs scores (nonparametric scale) in WT (C57BL/6) and P66Shc-/- mice have a less severe EAE disease course than WT mice. (b) EAE disease signs scores (nonparametric scale) in WT (C57BL/6) and Cyc-D-/- mice. (c) EAE disease signs scores (nonparametric scale) in WT (C57BL/6) and p66Shc/Cyc-D-/- mice. 
$48 \mathrm{~h}$ and were radiolabelled with $9,25 \mathrm{kBq} /$ well of methyl-3H thymidine in the last $16 \mathrm{~h}$ of coculture. The cells are harvested and counted by a beta radiation liquid scintillation counter.

\section{Results and Discussion}

3.1. P66Shc Determines Neuronal Cells Apoptosis. P66Shc expression has been already demonstrated in neuronal cell lines from different mammals [23]. We confirmed by Western Blot (WB) analysis the expression of p66Shc in several neuronal cell lines, in particular Kelly and SH-NY of human origin, and in the mouse spinal cord (Figure 1(a)). The phosphorylation of p66Shc at Serine 36 is the event activating the proapoptotic function of p66Shc $[8,16]$. WB analysis revealed p66Shc Ser36 phosphorylation in neuronal cell lines upon different challenges (Figure 1(b), [8]). Then, knocking down the p66Shc expression has been found to increase survival in Kelly cells (Figure 1(c)) as well as in other neuronal cells $[2,23]$ upon oxidative stress and other pro-apoptotic challenges. In agreement, the overexpression of p66Shc but not of the p66ShcSer36Ala mutant increased cell death in neuronal cells [23].

All these findings indicate that p66shc is present in neuronal cells, including human ones and confers sensitivity to apoptosis.

\subsection{P66Shc Does Not Affect Immune Response in EAE. EAE} is an acute or chronic-relapsing, demyelinating autoimmune disease model that closely resembles the human MS [24]. During EAE, as a consequence of specific myelin antigen experimental autoimmunization, activated T- and Blymphocytes infiltrate the central nervous system (CNS) where they produce large amounts of cytokines that together with activated microglia lead to demyelination and axonal degeneration $[25,26]$. Several evidences indicate that oligodendrocytes, the myelin-forming glial cells in the CNS, are sensitive to cell death stimuli such as cytotoxic cytokines, antimyelin antibodies, nitric oxide, and oxidative stress. Indeed, apoptosis has been established in glial cells of both human MS patients and EAE animal models, and it has been proposed to be the crucial mechanism of MS and EAE associated dysfunctions $[27,28]$.

To determine the role of p66Shc in EAE we compared the results of EAE in p66Shc-/- and WT mice. First, we immunized a total number of 9 isogenic WT and 9 p66Shc-lC57BL/6 mice with myelin oligodendrocyte glycoprotein (MOG) 35-55 peptide following standard protocols [29, 30]. At days 8, 10, and 36 upon MOG immunization, we sacrificed the mice ( 3 at each time) and extracted the blood and spleens to investigate anti-MOG antibodies titer and MOG-induced splenocyte proliferation, respectively. ELISA test using MOG coated wells revealed that the concentration of antibodies against MOG raised at the same time and extent in both WT and p66Shc-l- mice (Figure 2(a)). Likewise, thymidine incorporation as measure of splenocytes proliferation upon in vitro stimulation with MOG resulted to be not significantly altered by the deletion of p66Shc (Figure 2(b)). The secretion of TNF-alpha, IL-6, and interferon gamma by WT and
p66Shc-I- splenocytes was comparable as well in vitro (Figure 2(c)).

Therefore, p66Shc did not appear to influence humoral and cellular immune response to MOG immunization.

3.3. P66Shc Deletion Delays EAE. Then we performed EAE in WT and p66Shc-/- age matched female mice. Clinical score and weight were recorded daily using a nonparametric scale. Starting from 10 days (approximately depending on the different experiments) after MOG immunization, WT mice developed clinical symptoms, manifested as limb weakness and paralysis (WT score at day 10, 1,80 $\pm 0,3$, see Figure 3(a)) while p66Shc-1- mice did not (p66Shc-l- score at day 10, 0,0 see Figure 3(a)). The onset of the disease in p66Shc-lmice was significantly delayed (Figure 3(a) and Table 1). All over the experiment, the p66Shc-I- showed milder paralysis than WT and consistently a lower disease severity score than WT mice (Figure 3(a)).

Notably, the 20\% EAE WT mice died, whereas no p66Shc-/- died. P66Shc deletion was found to protect significantly from body weight loss as well (day 20 body weight of p66Shc-/- 19,20 $\pm 1,36$ versus WT 16,85 $\pm 1,13$ ).

3.4. Cyclophilin-D Deletion Did Not Change EAE Expression in p66Shc-/-Mice. To determine whether the protective effect of p66Shc deletion was mediated by PTP, we investigated the genetic interaction between the mutations of p66Shc and of Cyc-D that increases the opening threshold of PTP and was found to ameliorate EAE as well (Figure 3(b), [31]). Indeed, the exact molecular composition of the PTP is still debated [11]. However, some putative components have been indicated, such as the voltage-dependent anion channel (VDAC) located on the outer-mitochondrial membrane, the adenine nucleotide translocase (ANT, located on the inner mitochondrial membrane), and a matrix protein, Cyc-D, but results from knockout mice of these proteins confirmed only for Cyc-D a role in mitochondrial PT, although Cyc-D-/animals develop normally and undergo apoptosis in response to certain insults [32], suggesting that additional proteins are probably involved.

So, we generated p66Shc/Cyc-D-/- mice by crossing p66Shc-/- and Cyc-D-/- mice both in C57BL/6 background. Then, we performed EAE on the p66Shc/Cyc-D-/mice, and we evaluated the evolution of their disease over a month. The onset and development of EAE in p66Shc/CycD-/- mice resulted to be identical to those observed for p66Shc-/- mice (Figure 3(c) and Table 2). Notably, the early onset of disease typical of Cyc-D null mice (Figure 3(b), [31]) was lost when also p66Shc was mutated suggesting that p66Shc is necessary for the expression of disease before time by the Cyc-D mutation.

These results indicate that the deletion Cyc-D was not epistatic to the deletion of p66Shc with respect to the to EAE protection. 
TABLE 1: Clinical EAE parameters in WT and p66Shc-/- mice.

\begin{tabular}{|c|c|c|c|c|c|}
\hline Strain & Disease onset (day 10) score & Disease onset (day 13) score & Number of mice with score $\geq 3$ & Mean maximum score & AUC (days) \\
\hline WT & $1.80 \pm 0.3$ & $2.20 \pm 0.34$ & $3 / 5$ & $3.20 \pm 0.50$ & $21.95 \pm 3.54$ \\
\hline P66Shc-1- & 0 & $1.00 \pm 0.43$ & $2 / 5$ & $2.5 \pm 0.62$ & $13.65 \pm 4.91$ \\
\hline
\end{tabular}

Data are mean of three independent experiments \pm SE. (AUC: area under the curve) values are calculated at day 20 from the immunization.

TABLE 2: Clinical EAE parameters in WT, Cyc-D-/-, and p66Shc/Cyc-D-/- mice.

\begin{tabular}{|c|c|c|c|c|c|c|c|}
\hline Strain & $\begin{array}{l}\text { Disease onset } \\
\text { (day 11) score }\end{array}$ & $\begin{array}{l}\text { Disease onset } \\
\text { (day 13) score }\end{array}$ & $\begin{array}{l}\text { Survival at } \\
\text { sacrifice } \\
\text { Day } 27\end{array}$ & $\begin{array}{c}\begin{array}{c}\text { Number of } \\
\text { mice }\end{array} \\
\text { with score } \geq 3\end{array}$ & $\begin{array}{c}\text { Day of } \\
\text { maximal } \\
\text { score }\end{array}$ & $\begin{array}{c}\text { Mean } \\
\text { maximum } \\
\text { score }\end{array}$ & AUC (days) \\
\hline WT & $0.75 \pm 0.33$ & $2.45 \pm 0.18$ & $3 / 10$ & $10 / 10$ & 20 & $4.7 \pm 0.21$ & $22.10 \pm 2.91$ \\
\hline Cyc-D-/- & 0 & $2.08 \pm 0.30$ & $6 / 6$ & $4 / 6$ & 14 & $3.17 \pm 0.31$ & $26.83 \pm 2.55$ \\
\hline P66Shc/Cyc-D-/- & 0 & $1.14 \pm 0.37$ & $6 / 7$ & $5 / 7$ & 21 & $3.85 \pm 0.35$ & $23.50 \pm 2.24$ \\
\hline
\end{tabular}

Data are mean three independent experiments \pm SE. Values are calculated at day 20 from the immunization.

\section{Conclusions}

Neurodegenerative disease is complex trait disorder that involves several stress response pathways inducing mitochondrial apoptosis. Classical genetics studies, to identify susceptibility genes, are more difficult than for simple mendelian disorders because of the number of loci involved, the incomplete penetrance, and the important role of environmental factors.

Results form in vitro experiments showing that p66Shc increases oxidative stress to the mitochondria and the CycD-dependent PTP particularly supported the hypothesis of a genetic interaction of p66Shc and Cyc-D. So, to validate the role of the p66Shc-PTP pathway in neurodegeneration we have studied in mice the epistasis of p66Shc and Cyc-D null mutations on the susceptibility to EAE.

Results from these experiments revealed that both mutations mitigated the disease, but their effects were not additive. These findings indicate that the p66Shc and Cyc-D are epistatic and validate the hypothesis that p66Shc activation by stresses converges to the PTP opening to induce mitochondrial swelling in neurons.

Therefore, early detection of p66Shc activation (expression levels, specific phosphorylation) or its inhibition may represent valid approaches to treat neurodegeneration.

\section{Acknowledgments}

The authors thank Mike Forte of the Oregon Health and Science University, USA, and Paolo Bernardi of the University of Padova, Italy, for sharing the Cyc-D-/- mice, Maria Rescigno of the EIO and Iliyan D. Iliev for the experiment on T-cells, Mirella Trinei (EIO) for many helpful discussions, and Massimo Stendardo (EIO) for helping in taking care of the mice. This work was supported by National Institutes of Health Grant no. 1P01AG025532-01A1 awarded to PG.P.

\section{References}

[1] L. Luzi, S. Confalonieri, P. P. Di Fiore, and P. G. Pelicci, "Evolution of Shc functions from nematode to human," Current
Opinion in Genetics and Development, vol. 10, no. 6, pp. 668$674,2000$.

[2] E. Migliaccio, M. Giorgio, and P. G. Pelicci, "Apoptosis and aging: role of p66Shc redox protein," Antioxidants and Redox Signaling, vol. 8, no. 3-4, pp. 600-608, 2006.

[3] M. Trinei, I. Berniakovich, E. Beltrami et al., "P66Shc signals to age," Aging, vol. 1, no. 6, pp. 503-510, 2009.

[4] F. Orsini, E. Migliaccio, M. Moroni et al., "The life span determinant p66Shc localizes to mitochondria where it associates with mitochondrial heat shock protein 70 and regulates transmembrane potential," Journal of Biological Chemistry, vol. 279, no. 24, pp. 25689-25695, 2004.

[5] M. Giorgio, E. Migliaccio, F. Orsini et al., "Electron transfer between cytochrome $\mathrm{c}$ and p66Shc generates reactive oxygen species that trigger mitochondrial apoptosis," Cell, vol. 122, no. 2, pp. 221-233, 2005.

[6] Y. R. Kim, C. S. Kim, A. Naqvi et al., "Epigenetic upregulation of p66shc mediates low-density lipoprotein cholesterol-induced endothelial cell dysfunction," American Journal of Physiology, vol. 303, pp. H189-H196, 2012.

[7] M. Trinei, M. Giorgio, A. Cicalese et al., "A p53-p66Shc signalling pathway controls intracellular redox status, levels of oxidation-damaged DNA and oxidative stress-induced apoptosis," Oncogene, vol. 21, no. 24, pp. 3872-3878, 2002.

[8] P. Pinton, A. Rimessi, S. Marchi et al., "Protein kinase C $\beta$ and prolyl isomerase 1 regulate mitochondrial effects of the life-span determinant p66Shc," Science, vol. 315, no. 5812, pp. 659-663, 2007.

[9] F. Orsini, M. Moroni, C. Contursi et al., "Regulatory effects of the mitochondrial energetic status on mitochondrial p66Shc," Biological Chemistry, vol. 387, no. 10-11, pp. 1405-1410, 2006.

[10] M. Gertz, F. Fischer, D. Wolters, and C. Steegborn, "Activation of the lifespan regulator p66Shc through reversible disulfide bond formation," Proceedings of the National Academy of Sciences of the United States of America, vol. 105, no. 15, pp. 5705-5709, 2008.

[11] M. Forte and P. Bernardi, "Genetic dissection of the permeability transition pore," Journal of Bioenergetics and Biomembranes, vol. 37, no. 3, pp. 121-128, 2005.

[12] D. R. Green and G. Kroemer, "The pathophysiology of mitochondrial cell death," Science, vol. 305 , no. 5684, pp. 626-629, 2004 . 
[13] P. Bernardi, "Mitochondrial transport of cations: channels, exchangers, and permeability transition," Physiological Reviews, vol. 79, no. 4, pp. 1127-1155, 1999.

[14] W. Wang, H. Fang, L. Groom et al., "Superoxide flashes in single mitochondria," Cell, vol. 134, no. 2, pp. 279-290, 2008.

[15] R. Perez-Campo, M. López-Torres, S. Cadenas, C. Rojas, and G. Barja, "The rate of free radical production as a determinant of the rate of aging: evidence from the comparative approach," Journal of Comparative Physiology B, vol. 168, no. 3, pp. 149-158, 1998.

[16] C. Napoli, I. Martin-Padura, F. De Nigris et al., "Deletion of the p66Shc longevity gene reduces systemic and tissue oxidative stress, vascular cell apoptosis, and early atherogenesis in mice fed a high-fat diet," Proceedings of the National Academy of Sciences of the United States of America, vol. 100, no. 4, pp. 21122116, 2003.

[17] E. Migliaccio, M. Giogio, S. Mele et al., “The p66(shc) adaptor protein controls oxidative stress response and life span in mammals," Nature, vol. 402, no. 6759, pp. 309-313, 1999.

[18] S. Menini, L. Amadio, G. Oddi et al., "Deletion of p66Shc longevity gene protects against experimental diabetic glomerulopathy by preventing diabetes-induced oxidative stress," Diabetes, vol. 55, no. 6, pp. 1642-1650, 2006.

[19] K. G. Su, C. Savino, G. Marracci et al., "Genetic inactivation of the p66 isoform of ShcA is neuroprotective in a murine model of multiple sclerosis," European Journal of Neuroscience, vol. 35, pp. 562-571, 2012.

[20] A. Carpi, R. Menabò, N. Kaludercic, P. Pelicci, F. Di Lisa, and M. Giorgio, "The cardioprotective effects elicited by p66Shc ablation demonstrate the crucial role of mitochondrial ROS formation in ischemia/reperfusion injury," Biochimica et Biophysica Acta, vol. 1787, no. 7, pp. 774-780, 2009.

[21] I. Berniakovich, M. Trinei, M. Stendardo et al., "p66Shcgenerated oxidative signal promotes fat accumulation," Journal of Biological Chemistry, vol. 283, no. 49, pp. 34283-34293, 2008.

[22] A. Berry, F. Capone, M. Giorgio et al., "Deletion of the life span determinant p66Shc prevents age-dependent increases in emotionality and pain sensitivity in mice," Experimental Gerontology, vol. 42, no. 1-2, pp. 37-45, 2007.

[23] M. Cozzolino, M. G. Pesaresi, V. Gerbino, J. Grosskreutz, and M. T. Carrì, "Amyotrophic lateral sclerosis: new insights into underlying molecular mechanisms and opportunities for therapeutic intervention," Antioxidants \& Redox Signaling, vol. 17, pp. 1277-1330, 2012.

[24] G. Martino, L. Adorini, P. Rieckmann et al., "Inflammation in multiple sclerosis: the good, the bad, and the complex," Lancet Neurology, vol. 1, no. 8, pp. 499-509, 2002.

[25] L. Steinman, "Engineering better cytokines," Nature Biotechnology, vol. 21, no. 11, pp. 1293-1294, 2003.

[26] L. Steinman, "Immune therapy for autoimmune diseases," Science, vol. 305, no. 5681, pp. 212-216, 2004.

[27] E. Julià, M. C. Edo, A. Horga, X. Montalban, and M. Comabella, "Differential susceptibility to apoptosis of CD4+T cells expressing CCR5 and CXCR3 in patients with MS," Clinical Immunology, vol. 133, no. 3, pp. 364-374, 2009.

[28] M. Irony-Tur-Sinai, N. Grigoriadis, D. Tsiantoulas, O. Touloumi, O. Abramsky, and T. Brenner, "Immunomodulation of EAE by alpha-fetoprotein involves elevation of immune cell apoptosis markers and the transcription factor FoxP3," Journal of the Neurological Sciences, vol. 279, no. 1-2, pp. 80-87, 2009.
[29] S. Amor, J. K. O’Neill, M. M. Morris et al., "Encephalitogenic epitopes of myelin basic protein, proteolipid protein, and myelin oligodendrocyte glycoprotein for experimental allergic encephalomyelitis induction in Biozzi $\mathrm{ABH}(\mathrm{H}-2 \mathrm{Ag} 7)$ mice share an amino acid motif," Journal of Immunology, vol. 156, no. 8, pp. 3000-3008, 1996.

[30] R. Furlan, E. Brambilla, F. Ruffini et al., "Intrathecal delivery of IFN- $\gamma$ protects C57BL/ 6 mice from chronic-progressive experimental autoimmune encephalomyelitis by increasing apoptosis of central nervous system-infiltrating lymphocytes," Journal of Immunology, vol. 167, no. 3, pp. 1821-1829, 2001.

[31] M. Forte, B. G. Gold, G. Marracci et al., "Cyclophilin $\mathrm{D}$ inactivation protects axons in experimental autoimmune encephalomyelitis, an animal model of multiple sclerosis," Proceedings of the National Academy of Sciences of the United States of America, vol. 104, pp. 7558-7563, 2007.

[32] E. Basso, L. Fante, J. Fowlkes, V. Petronilli, M. A. Forte, and P. Bernardi, "Properties of the permeability transition pore in mitochondria devoid of cyclophilin D," Journal of Biological Chemistry, vol. 280, no. 19, pp. 18558-18561, 2005. 


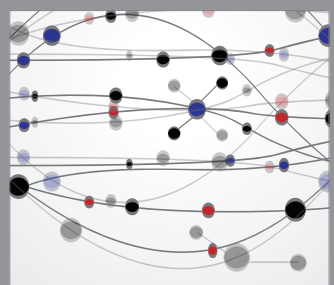

The Scientific World Journal
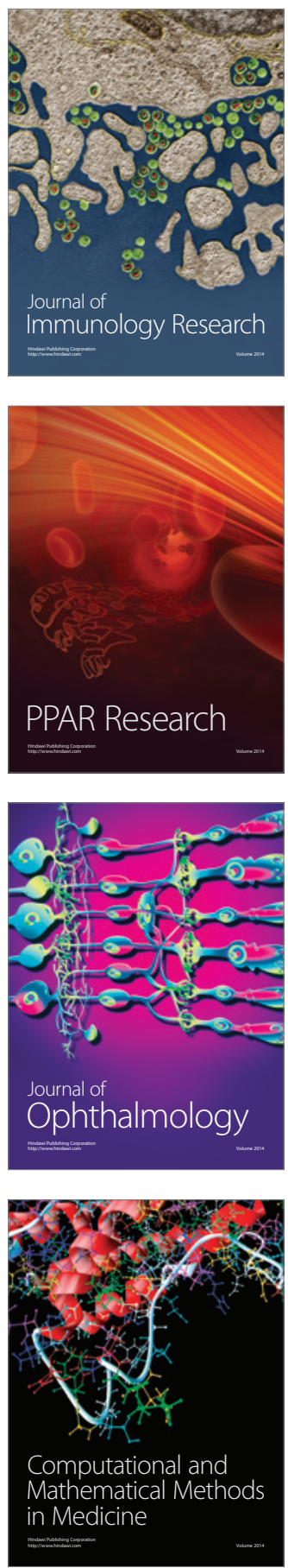

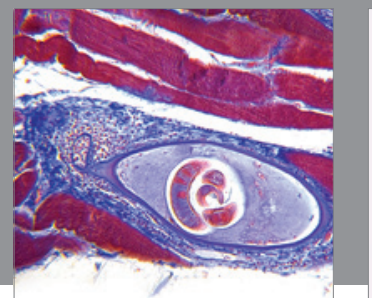

Gastroenterology

Research and Practice
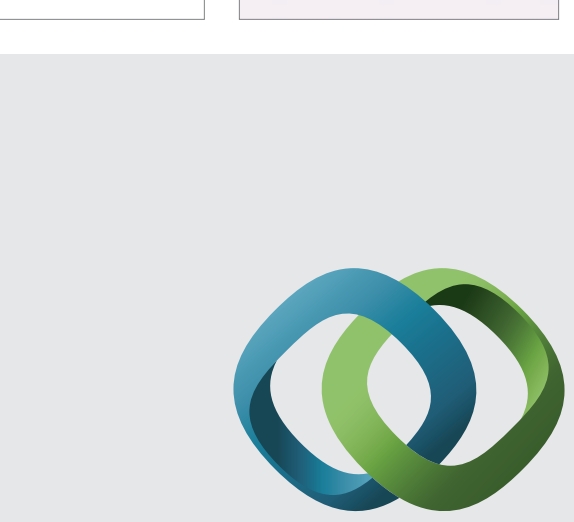

\section{Hindawi}

Submit your manuscripts at

http://www.hindawi.com
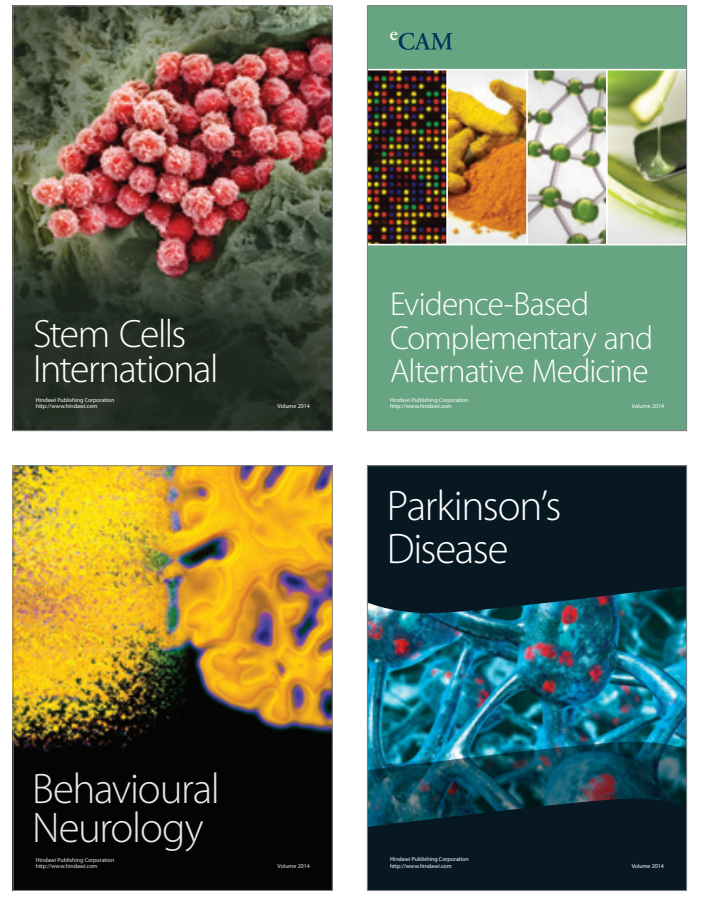
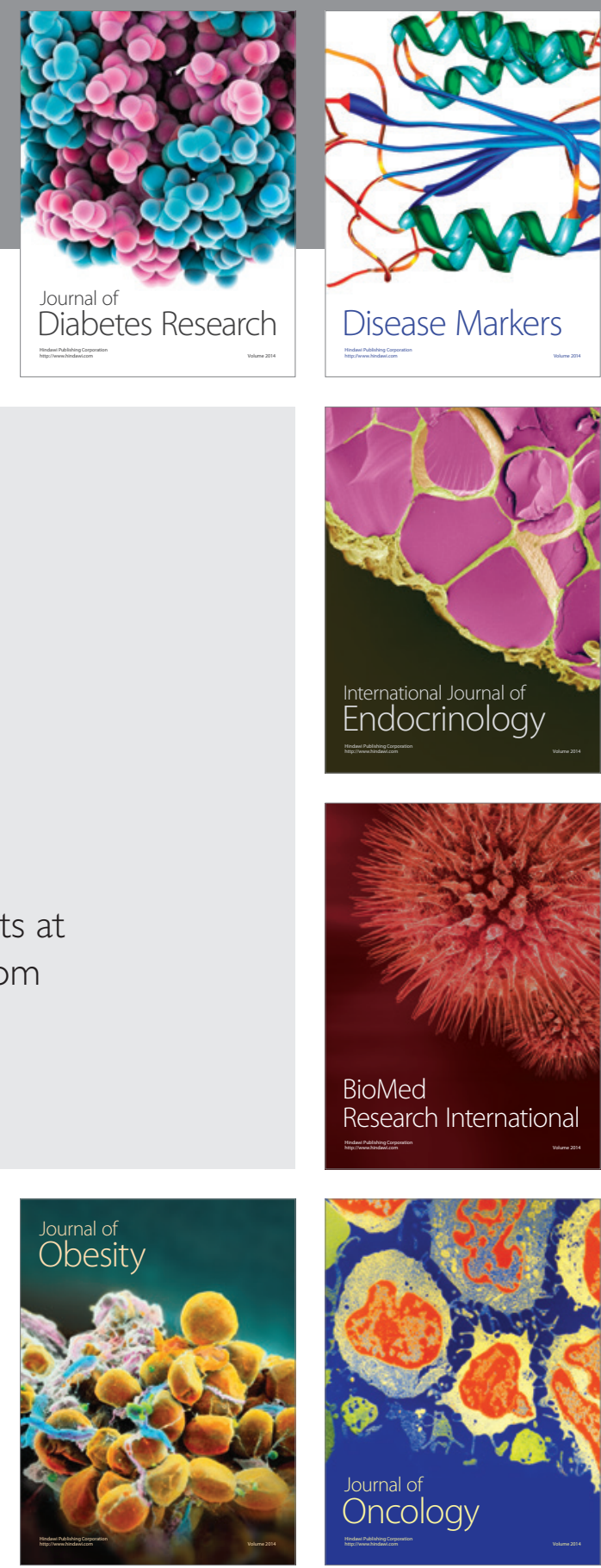

Disease Markers
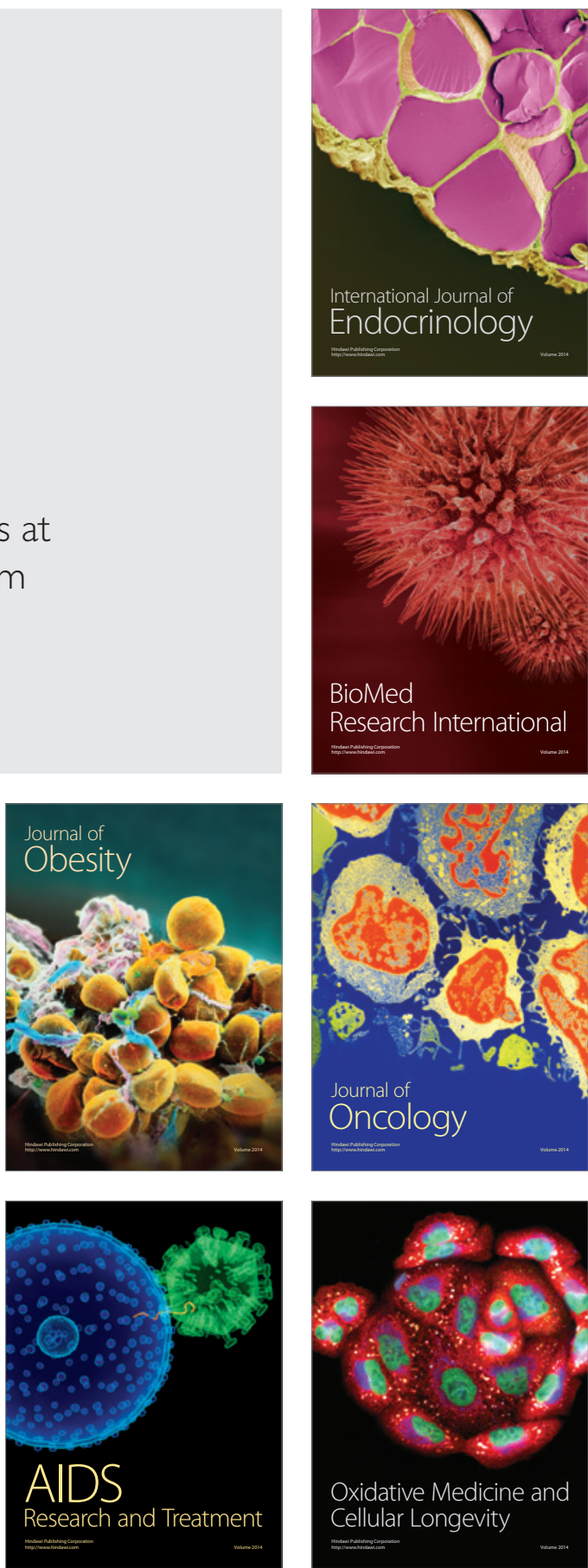